\title{
Describing the Brain in Autism in Five Dimensions-Magnetic Resonance Imaging-Assisted Diagnosis of Autism Spectrum Disorder Using a Multiparameter Classification Approach
}

\author{
Christine Ecker, ${ }^{1}$ Andre Marquand, ${ }^{2}$ Janaina Mourão-Miranda,,${ }^{3,4}$ Patrick Johnston, ${ }^{1}$ Eileen M. Daly, ${ }^{1}$ \\ Michael J. Brammer, ${ }^{2}$ Stefanos Maltezos, ${ }^{1}$ Clodagh M. Murphy, ${ }^{1}$ Dene Robertson, ${ }^{1}$ Steven C. Williams, ${ }^{3}$ \\ and Declan G. M. Murphy ${ }^{1}$ \\ ${ }^{1}$ Section of Brain Maturation, Department of Psychological Medicine, Institute of Psychiatry, ${ }^{2}$ Brain Image Analysis Unit, Department of Biostatistics, \\ Institute of Psychiatry, and ${ }^{3}$ Centre for Neuroimaging Sciences, Institute of Psychiatry, King's College, London SE5 8AF, United Kingdom, and ${ }^{4}$ Centre for \\ Computational Statistics and Machine Learning, Department of Computer Science, University College London, London WC1E 6BT, United Kingdom
}

Autism spectrum disorder (ASD) is a neurodevelopmental condition with multiple causes, comorbid conditions, and a wide range in the type and severity of symptoms expressed by different individuals. This makes the neuroanatomy of autism inherently difficult to describe. Here, we demonstrate how a multiparameter classification approach can be used to characterize the complex and subtle structural pattern of gray matter anatomy implicated in adults with ASD, and to reveal spatially distributed patterns of discriminating regions for a variety of parameters describing brain anatomy. A set of five morphological parameters including volumetric and geometric features at each spatial location on the cortical surface was used to discriminate between people with ASD and controls using a support vector machine (SVM) analytic approach, and to find a spatially distributed pattern of regions with maximal classification weights. On the basis of these patterns, SVM was able to identify individuals with ASD at a sensitivity and specificity of up to $90 \%$ and $80 \%$, respectively. However, the ability of individual cortical features to discriminate between groups was highly variable, and the discriminating patterns of regions varied across parameters. The classification was specific to ASD rather than neurodevelopmental conditions in general (e.g., attention deficit hyperactivity disorder). Our results confirm the hypothesis that the neuroanatomy of autism is truly multidimensional, and affects multiple and most likely independent cortical features. The spatial patterns detected using SVM may help further exploration of the specific genetic and neuropathological underpinnings of ASD, and provide new insights into the most likely multifactorial etiology of the condition.

\section{Introduction}

Autism spectrum disorder (ASD) is a highly heterogeneous neurodevelopmental condition with multiple causes and courses, a wide range in symptom severity, and several associated comorbid disorders (Amaral et al., 2008). It has thus been suggested that ASD should be thought of as "the autisms" rather than a single autistic phenotype (Geschwind, 2007; Geschwind and Levitt, 2007). This makes the neuroanatomy of autism inherently difficult to describe.

Although several autistic "core" structures have repeatedly been highlighted by previous studies, such as abnormalities in frontal, parietal, and limbic regions, as well as the basal ganglia and the cerebellum [e.g., McAlonan et al. (2002), Waiter et al.

\footnotetext{
Received Nov. 2, 2009; revised May 7, 2010; accepted June 8, 2010.

This work was undertaken with the support of the A.I.M.S. Consortium (Autism Imaging Multicentre Study), which is funded by the Medical Research Council UK (G0400061), and a programme grant on the diagnosis and treatment of young adults with autism funded by the National Institute for Health Research (RP-PG-0606-1045). We would also like to thank the Wellcome Trust, which funded J.M.-M.

This article is freely available online through the J Neurosci Open Choice option.

Correspondence should be addressed to Dr. Christine Ecker, Section of Brain Maturation, P050, Institute of Psychiatry, De Crespigny Park, London SE5 8AF, UK. E-mail: Christine.ecker@kcl.ac.uk.

DOI:10.1523/JNEUROSCI.5413-09.2010

Copyright $\odot 2010$ the authors $\quad$ 0270-6474/10/3010612-12\$15.00/0
}

(2004), McAlonan et al. (2005), and Rojas et al. (2006)], reports of region-specific differences in ASD are highly variable [for review, see Toal et al. (2005) and Amaral et al. (2008)]. Such variable findings may simply be explained by confounds such as clinical heterogeneity between studies, or analytical techniques. Alternatively, variability in findings may indicate that differences in brain anatomy in ASD are relatively subtle and spatially distributed, and are difficult to detect using mass-univariate (i.e., voxelwise) approaches. Last, given the multiple etiology of ASD, it is likely that its neuroanatomy is not confined to a single morphological parameter but affects multiple cortical features.

There is already evidence to suggest that several aspects of cerebral morphology are different in people with ASD_-including both volumetric (i.e., cortical thickness, regional area) and geometric (i.e., cortical shape) features (Levitt et al., 2003; Nordahl et al., 2007); and that different morphological features may have different neuropathological and genetic underpinnings (Panizzon et al., 2009). For instance, cortical thickness is likely to reflect dendritic arborization (Huttenlocher, 1990), while cortical surface area has been linked to the number of minicolumns in the cortical layer (Rakic, 1988). Geometric features such as cortical folding pattern, on the other hand, may reflect an abnormal pattern of intrinsic as well as extrinsic con- 
Table 1. Subject demographics

\begin{tabular}{lcc}
\hline & ASD $(n=20)$ & Controls $(n=20)$ \\
\hline Age, years & $33 \pm 11(20-68)$ & $36 \pm 9(20-49)$ \\
Full-scale IQ & $103 \pm 20(76-141)$ & $110 \pm 13(77-129)$ \\
Verbal IQ $^{*}$ & $102 \pm 17(78-133)$ & $106 \pm 14(71-131)$ \\
Performance IQ $^{*}$ & $98 \pm 19(77-138)$ & $110 \pm 14(84-136)$ \\
ADI-R social $^{a}$ & $15 \pm 4$ & - \\
ADI-R communication $^{a}$ & $10 \pm 3$ & - \\
ADI-R repetitive behavior $^{a}$ & $4 \pm 2$ & - \\
ADOS total $^{b}$ & $10 \pm 2$ & -
\end{tabular}

Data are expressed as mean \pm SD (range). There were no significant differences between subject groups in age and

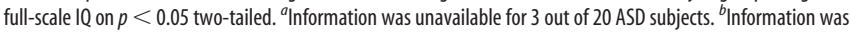
available for five ASD subjects; two cases had both ADOS and ADI. * Significant on $p<0.05$.

nectivity (Van Essen, 1997). Thus, examining the relationship between such multiple cortical features could provide invaluable insights into the multifactorial etiology of ASD.

So far, individual aspects of the cortex are generally explored in isolation (i.e., in separate statistical models). Hence it is not yet known (1) whether they equally contribute to differentiating individuals with ASD from controls; and (2) what the relationship between such multiple cortical abnormalities in ASD is. In the present study, we therefore used a multiparameter classification approach using a support vector machine (SVM) [e.g., MourãoMiranda et al. (2005), Davatzikos et al. (2008), and Klöppel et al. (2008)] to investigate brain anatomy in adults with ASD. Here, a variety of morphological features, including both volumetric and geometric parameters, were used simultaneously to classify control and ASD individuals, as well as a neurodevelopmental control group of individuals with attention deficit hyperactivity disorder (ADHD). We aimed to demonstrate that the neuroanatomical patterns discriminating individuals with autism from controls are truly multidimensional, comprising multiple and most likely independent cortical features. If so, this may guide further exploration of the specific genetic and neuropathological underpinnings of ASD.

\section{Materials and Methods}

Participants. Twenty control adults were recruited locally by advertisement and 20 adults with ASD were recruited through a clinical research program at the Maudsley Hospital/Institute of Psychiatry (London). All volunteers (see Table 1) gave informed consent (as approved by the Institute of Psychiatry and Bethlem and Maudsley Hospital Trust research ethics committee), and had a full-scale intelligence quotient (FSIQ) $>75$ [WASI (Wechsler, 1999)]. All volunteers were right-handed males, and were between 20 and 68 years of age. None had a history of major psychiatric disorder or medical illness affecting brain function (e.g., psychosis or epilepsy). All participants underwent a psychiatric interview and physical examination. Blood tests were used to exclude biochemical, hematological, or chromosomal abnormalities (including fragile X syndrome). All participants with ASD were diagnosed with autism according to ICD 10 research criteria (World Health Organization, 1992) by a trained and qualified clinician in our Adult Autism Specialist Clinic at the South London and Maudsley Hospital. The initial clinical diagnosis was then confirmed using the Autism Diagnostic Interview-Revised [ADI-R (Lord et al., 1994)] whenever possible $(n=$ 17); that is, where parents agreed to take part. In three out of the 20 included cases, ADI scores could not be obtained due to informants being unavailable. In these three cases, the clinical diagnosis was confirmed using the Autism Diagnostic Observation Schedule [ADOS (Lord et al., 1989)]. Both ADI-R and ADOS scores were available for two participants. All cases had to reach the ADOS or ADI algorithm cutoffs in the three domains of impaired reciprocal social interaction, communication and repetitive behaviors and stereotyped patterns, but we did allow failure to reach cutoff in one of the domains (by one point). Sixteen ASD individuals did not have a delay in development of phrase speech at the age of 36 months (and so may be subtyped by some as having the autistic subtype of Asperger's syndrome); the remaining four individuals had a history of delayed phrase speech (and so may be subtyped by some as having the autistic subtype of high functioning autism). However, due to the small sample size it was not possible to reliably investigate putative differences between people with high functioning autism and Asperger's syndrome, and all subjects were analyzed in a combined group of individuals with ASD.

Furthermore, we recruited a group of 19 individuals with ADHD from the Adult ADHD services at the Maudsley Hospital, London. This group served as a neurodevelopmental control group and was matched to the ASD group in gender, age (mean $\pm \mathrm{SD}=31 \pm 8.5)$, FSIQ (mean $\pm \mathrm{SD}=$ $107 \pm 16$ ), and handedness. Of these, eight individuals fulfilled the criteria for ADHD combined type and 11 for ADHD inattentive type. The diagnosis of ADHD was made by a trained and qualified clinician based on a structured clinical interview according to DSM-IV criteria. ADHD symptoms were also assessed using Barkley Current and Childhood Symptoms Scales (Barkley, 2006) and the Wender-Utah Rating Scale [WURS-25 (Ward et al., 1993)].

MRI data acquisition. MRI data were acquired using a 1.5 T GE Signa Neuro-optimized System (General Electric Medical Systems) fitted with $40 \mathrm{mT} / \mathrm{m}$ high-speed gradients at the Maudsley Hospital, London. Whole-brain spoiled gradient recalled acquisition in the steady-state T1weighted series were collected in the coronal plane with repetition time $=$ $13.8 \mathrm{~ms}$, echo time $=2.8 \mathrm{~ms}$, yielding 124 contiguous $1.5 \mathrm{~mm}^{2}$ axial slices of $256 \times 192$ voxels with an in-plane resolution of $1 \mathrm{~mm}^{2}$. Similar T1weighted MRI scans were acquired for the ADHD group on a 1.5 T GE Signa Neuro-optimized System at the Maudsley Hospital, London, using repetition time $=10.72 \mathrm{~ms}$, echo time $=4.86 \mathrm{~ms}$, yielding 146 contiguous $1.1 \mathrm{~mm}^{2}$ axial slices. A quadrature birdcage head coil was used for radio-frequency transmission and reception. Foam padding and a forehead strap were used to limit head motion. All scans were visually inspected by a radiologist to assess (1) image contrast, (2) movement artifacts, and (3) the existence of clinical abnormalities. Scans displaying low image quality or clinical abnormalities were excluded from this study.

Image processing. Cortical reconstruction and volumetric segmentation were performed with the Freesurfer image analysis suite, which is documented and freely available for download online (http://surfer.nmr. mgh.harvard.edu/). The technical details of these procedures are described in prior publications (Dale et al., 1999; Fischl et al., 1999, 2002, 2004a,b; Fischl and Dale, 2000; Ségonne et al., 2004; Jovicich et al., 2006). Briefly, this processing includes motion correction and averaging of multiple volumetric T1-weighted images (when more than one is available), removal of nonbrain tissue using a hybrid watershed/surface deformation procedure (Ségonne et al., 2004), automated Talairach transformation, segmentation of the subcortical white matter and deep gray matter volumetric structures (including hippocampus, amygdala, caudate, putamen, and ventricles) (Fischl et al., 2002, 2004b), intensity normalization, tessellation of the gray matter white matter boundary, automated topology correction (Fischl et al., 2001; Ségonne et al., 2007), and surface deformation following intensity gradients to optimally place the gray/ white and gray/CSF borders at the location where the greatest shift in intensity defines the transition to the other tissue class (Dale et al., 1999). Once the cortical models are complete, a number of deformable procedures were performed for further data processing and analysis, including surface inflation (Dale et al., 1999), registration to a spherical atlas, which used individual cortical folding patterns to match cortical geometry across subjects (Fischl et al., 1999), parcellation of the cerebral cortex into units based on gyral and sulcal structure (Fischl et al., 2004a; Desikan et al., 2006), and creation of a variety of surface-based data, including maps of curvature and sulcal depth (see below). This method uses both intensity and continuity information from the entire three-dimensional MR volume in segmentation and deformation procedures to produce representations of cortical thickness, calculated as the closest distance from the gray/white boundary to the gray/CSF boundary at each vertex on the tessellated surface (Fischl and Dale, 2000). The maps are created using spatial intensity gradients across tissue classes and are therefore not simply reliant on absolute signal intensity. The maps produced are not re- 
stricted to the voxel resolution of the original data thus are capable of detecting submillimeter differences between groups. Procedures for the measurement of cortical thickness have been validated against histological analysis (Rosas et al., 2002) and manual measurements (Kuperberg et al., 2003; Salat et al., 2004). Freesurfer morphometric procedures have been demonstrated to show good test-retest reliability across scanner manufacturers and across field strengths (Han et al., 2006). All reconstructed surfaces were visually inspected for gross-anatomical topological defects. Brains that did not reconstruct or showed geometric inaccuracies were excluded from the study.

A set of five morphometric parameters per vertex were used as input to the multimodal classifier. Three parameters [1-3: average convexity or concavity, mean (radial) curvature, and metric distortion] accounted for geometric features at each cerebral vertex and two parameters $(4,5$ : cortical thickness and surface area, respectively) measured volumetric features.

(1) The average convexity or concavity $(\boldsymbol{C})$ was used for quantifying the primary folding pattern of a surface. $C$ captures large-scale geometric features and is insensitive to noise in the form of small wrinkles in a surface (Fischl et al., 1999). At each vertex, $C$ indicates the depth/ height above the average surface and measures sulcal depth or gyral height, respectively. Differences in sulcal depth have previously been investigated as one important aspect of the cerebral geometry implicated in ASD (Nordahl et al., 2007), and have been suggested to reflect abnormal pattern of cortical connectivity.

(2) Mean (radial) curvature was used to assess folding of the small secondary and tertiary folds in the surface. The selection of this feature was motivated by early findings of polymicrogyria (i.e., excessive number of small convolutions on the surface of the brain) in autism (Piven et al., 1990). These would not be captured by the average convexity measure reflecting large-scale geometric features only.

(3) Metric distortion (i.e., Jacobian) indicating the degree of cortical folding was calculated as degree of displacement and convolution of the cortical surface relative to the average template (Fischl et al., 1999; Wisco et al., 2007). While sulcal depth and radial curvature measure specific aspects of the cortical geometry, metric distortion is a wider measure of the overall degree of cortical folding, and thus captures geometric distortions otherwise not specified. There is a large body of evidence to suggest that individuals with ASD display abnormal patterns of cortical gyrification reflecting cerebral development and connectivity [e.g., Levitt et al. (2003) and Hardan et al. (2004)].

(4) Cortical thickness (see above) and (5) pial area (i.e., the area of a vertex on the gray matter surface, calculated as the average of the area of the triangles touching that vertex) were used to quantify volumetric differences. Although these two features are generally combined to measure regional brain volume, a recent study has shown that cortical thickness and surface are influenced by distinct genetic mechanism (Panizzon et al., 2009), and we hence added as separate features into the model. A summary of the set of parameters is displayed in Figure 1.

Group differences in intracranial volume, total brain volume, and gray matter volume as estimated by FreeSurfer were assessed using $t$ tests for independent samples before classification. There were no significant differences between groups in any of these parameters at a level of $p<0.05$ (see Table 2). There were also no significant differences in total brain volume between controls and individuals with ADHD, nor between ASD and ADHD group on $p<0.05$. Bartlett's test for homogeneity of variances was used to examine parameter variability across homolog regions in different hemispheres using a threshold of $p<0.0014$ (corrected for multiple comparisons).
B Cortical Thickness
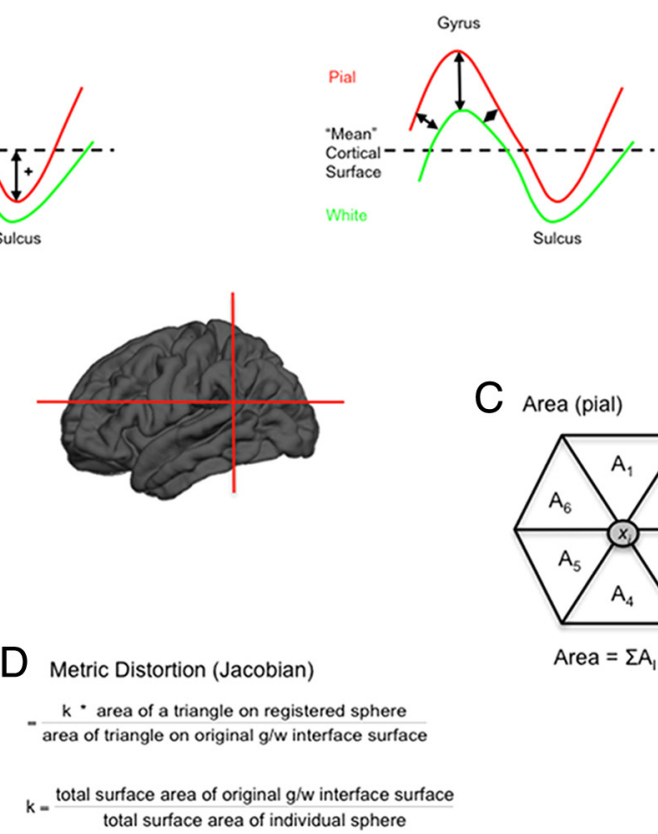

C Area (pial)

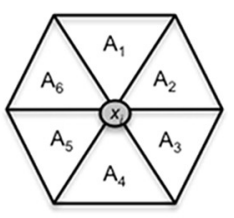

Area $=\Sigma A_{1} / 6$

Classification and support vector machine. A linear SVM was used to classify between patient and control group on the basis of their brain morphology. SVM has previously been applied to MRI data (MourãoMiranda et al., 2005; Davatzikos et al., 2008; Klöppel et al., 2008), and a detailed description was given by Burges (1998) and Schoelkopf and Smola (2002). Briefly, SVM is a supervised multivariate classification method that treats each image as a point in a high dimensional space. If SVM is applied to images coming from different modalities, as in the current study, the number of dimensions equals the number of voxels/ vertices per image multiplied by the number of modalities. Input data were then classified into two classes (e.g., individuals with ASD and controls) by identifying a separating hyperplane or decision boundary. The algorithm is initially trained on a subset of the data $\langle\boldsymbol{x}, \mathrm{c}\rangle$ to find a hyperplane that best separates the input space according to the class labels $c$ (e.g., -1 for patients, +1 for controls). Here, $x$ represents the input data (i.e., feature vector). The feature vector was generated by concatenating the five image modalities for each subject. Once the decision function is learned from the training data, it can be used to predict the class of a new test example. SVM (Vapnik, 1995) is a maximum margin classifier, which identifies the optimal hyperplane by finding the hyperplane with the maximum margin (i.e., maximal separation between classes). The margin is the distance from the separating hyperplane to the closest training examples. The training examples that lie on the margin are called support vectors. The hyperplane is defined by a weight vector and an offset. The weight vector is a linear combination of the support vectors and is normal to the hyperplane. The linear kernel SVM used in the present study allows direct extraction of the weight vector as an image 
Table 3. Results of SVM classification between ASD and control group using different brain morphometric features in the left and right hemispheres

\begin{tabular}{lllll}
\hline Morphometric feature & Correctly classified (\%) & Sensitivity (\%) & Specificity (\%) & $p$ \\
\hline Left hemisphere & & & & \\
$\quad$ All parameters & 85 & 90 & 80 & $0^{*}$ \\
Cortical thickness & 90 & 90 & 90 & $0^{*}$ \\
Radial curvature & 72.5 & 65 & 80 & $<0.001$ \\
Average convexity & 70 & 75 & 65 & $<0.004$ \\
Metric distortion & 80 & 80 & 80 & $0^{*}$ \\
$\quad$ Pial area & 77.5 & 70 & 85 & $0^{*}$ \\
Right hemisphere & & & & \\
All parameters & 65 & 60 & 70 & $<0.03$ \\
Cortical thickness & 60 & 65 & 55 & $<0.01$ \\
Radial curvature & 52.5 & 50 & 55 & $<0.30$ \\
Average convexity & 50 & 40 & 60 & $<0.40$ \\
Metric distortion & 57.5 & 45 & 70 & $<0.06$ \\
Pial area & 45 & 45 & 45 & $<0.60$ \\
\hline
\end{tabular}

Correctly identified ASD cases were considered true positive. * $p$ values of zero indicate that not a single one of the 1000 permutations provided a better classification.

with the labels +1 and -1 , respectively, a positive value in the discrimination map (red/yellow color scale) indicates relatively higher parameter values in patients than in controls with respect to the hyperplane, and a negative weight (blue/cyan color scale) means relatively higher parameter values in controls than in patients with respect to the hyperplane. Because the classifier is multivariate by nature, the combination of all voxels as a whole is identified as a global spatial pattern by which the groups differ (i.e., the discriminating pattern). To enable visualization of the discriminating pattern for each image modality, the weight vector was cut into its constituent parts, which were then mapped back onto the average white matter surface. In the present study, we colored all voxels that have values higher than $30 \%$ of the maximum value of the discrimination map (Mourão-Miranda et al., 2005, 2006). This threshold, although ultimately arbitrary, eliminates noise components predominantly $(<30 \%)$, thus enabling a better visualization of the most discriminating regions (31-100\%).

Intraregional morphometric profiles. To identify the relative contribution of specific parameters in discriminating between groups at different locations on the cortical surface, we displayed so called intraregional morphometric profiles. These profiles were derived by calculating the average weights of vertices within regions of interest (ROIs) for the five different parameters. ROIs were based on contiguous weight clusters from the overall discrimination maps. The choice of the ROIs, although ultimately arbitrary, was motivated by (1) the relevance of a region to the current literature [e.g., sulcal depth differences in intraparietal sulcus (Nordahl et al., 2007)], and (2) generally high parameter weights for a specific morphometric feature (e.g., high weights for cortical thickness in medial temporal sulcus). The ROI analysis aimed to illustrate that different regions can display distinct differences in one or more parameters, rather than displaying the same pattern of feature weights. As all weights were scaled, means and SDs are directly comparable across regions and parameters.

Cross-validation and permutation testing. The performance of the classifier was validated using the commonly used leave-two-out crossvalidation approach. This validation approach provides robust parameter estimates particularly for smaller samples. In each trial observations from all but one subject from each group were used to train the classifier. Subsequently, the class assignment of the test subjects was calculated during the test phase. This procedure was repeated $S=20$ times (where $S$ is the number of subjects per group), each time leaving observations from a different subject from each group out. The accuracy of the classifier was measured by the proportion of observations that were correctly classified into patient or control group. We also quantified the sensitivity and specificity of the classifier defined as Sensitivity $=\mathrm{TP} /(\mathrm{TP}+\mathrm{FN})$ and Specificity $=$ $\mathrm{TN} /(\mathrm{TN}+\mathrm{FP})$, where $\mathrm{TP}$ is the number of true positives, i.e., the number of patient images

(i.e., the SVM discrimination map). A parameter $C$, which controls the trade-off between having zero training errors and allowing misclassifications, was fixed at $C=1$ for all cases (default value). The LIBSVM toolbox for Matlab was used to perform the classifications (http://www.csie.ntu.edu.tw/ cjlin/libsvm/).

Discrimination maps. The weight vector has the same dimension as the feature vector and is normal to the hyperplane. It can be thought of as a spatial representation of the decision boundary, and thus represents a map of the most discriminating regions. Here, the feature vector had $n \times$ $m$ dimensions, where $n$ equals the number of vertices and $m$ denotes the number of modalities (i.e., five). Given two groups (ASD vs controls), correctly classified; $\mathrm{TN}$ is the number of true negatives, i.e., number of control images correctly classified; FP is the number of false positives, i.e., number of controls images classified as patients; and FN is the number of false negatives, i.e., number of patients images classified as controls.

Classifications were made first, by including all five morphological modalities into the feature vector, and, second, on the basis of each individual parameter. Different classifiers were trained for each hemisphere. This enabled us to assess the overall classification accuracies for individual parameters and hemispheres. Classifier performance was evaluated using basic receiver operating characteristics (ROC) graphs as well 
as permutation testing. Permutation testing can be used to evaluate the probability of getting specificity and sensitivity values higher than the ones obtained during the cross-validation procedure by chance. We permuted the labels 1000 times without replacement, each time randomly assigning patient and control labels to each image and repeated the crossvalidation procedure. We then counted the number of times the specificity and sensitivity for the permuted labels were higher than the ones obtained for the real labels. Dividing this number by 1000 we derived a $p$ value for the classification.

Last, the established classifier was used to predict group membership of individuals with ADHD. To achieve this, the classifier was first retrained using all ASD-control pairs to provide the best possible prediction model for discriminating the two groups. This trained classifier was then applied to the multiparameter data from the ADHD group and predictions for group membership for these subjects were derived.

To validate the binary classification of the subject groups on a quantitative level, and to identify the degree to which the classification is driven by autistic symptoms rather than confounds unrelated to autism, the test margin for each subject coming from the all included classifier was correlated with the level of symptom severity measured by the ADI subscales. A similar approach has previously been employed by Ecker et al. (2010).

\section{Results}

\section{Overall classifier performance}

Classification accuracies as well as sensitivity and specificity for each classifier are listed in Table 3. On the whole, strong hemispheric asymmetry was observed with regards to the overall classification accuracy. The left hemisphere provided consistently higher and above chance prediction accuracies across all morphometric parameters. Here, individuals with ASD were correctly assigned to the appropriate diagnostic category in $85.0 \%$ of all cases when all parameters were considered simultaneously (Fig. 2C). The sensitivity of the multiparameter classification in the left hemisphere was $90.0 \%$; i.e., if a volunteer had a clinical diagnosis of ASD, the probability that this participant was correctly assigned to the ASD category was 0.9 . The specificity was $80.0 \%$ - meaning that $80.0 \%$ of the controls subjects were correctly classified as controls. High classification accuracies were also obtained when individual morphological parameters were considered. Figure $2 \mathrm{~A}$ shows the receiver operator characteristics or ROC graph for the six classifiers in the left hemisphere. In general, one point in ROC space is better than another if it is to the northwest (TP rate is high, FP rate is low, or both) with the point $(0,1)$ representing perfect classification. Classifiers appearing on the left-hand side of the ROC graph, near the $x$-axis, may be considered "conservative" (i.e., make positive classifications only with strong evidence but often have low TP rates), whereas classifiers on the upper right-hand side of the graph may be thought of as "liberal" (i.e., make positive classifications with weak evidence so they classify nearly all positives correctly but often have high FPs). The diagonal line $y=x$ represents the strategy of randomly guessing a class. Best discrimination was obtained when cortical thickness measures were used to classify between groups with sensitivity and specificity values as high as $90.0 \%$, followed by a classification on the basis of the metric distortion parameter (sensitivity $80.0 \%$, specificity $80.0 \%$ ). The average convexity, pial area, and mean curvature displayed accu-
Table 4. Correlation coefficients between ADI diagnostic criteria and weight vector for the model combining all parameters

\begin{tabular}{llllll}
\hline & \multicolumn{2}{l}{ Left hemisphere } & & \multicolumn{2}{l}{ Right hemisphere } \\
\cline { 2 - 3 } Diagnostic test $(n=17)$ & $r$ & $p$ & & $r$ & $p$ \\
\hline ADI-R social & $0.414^{*}$ & $<0.04$ & & -0.152 & $<0.28$ \\
ADI-R communication & $0.620^{* *}$ & $<0.01$ & & -0.074 & $<0.38$ \\
ADI-R repetitive behavior & 0.161 & $<0.26$ & & -0.198 & $<0.22$ \\
\hline
\end{tabular}

* denotes significant correlation on $p<0.05$ (1-tailed); ${ }^{* *}$ denotes significant correlation on $p<0.01$ (1-tailed).
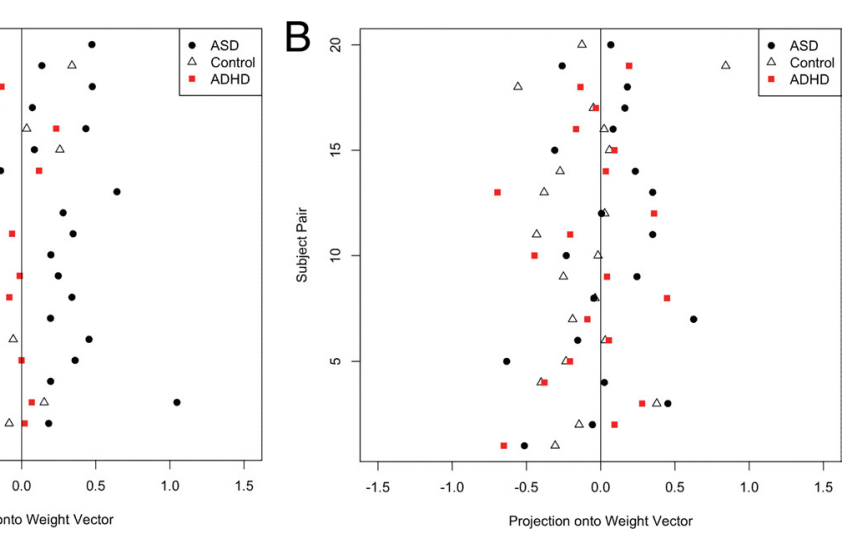

Prection onto Weight Vector

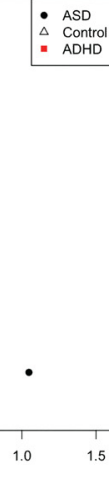

Figure 3. Classification plots showing group allocation of individuals with ADHD (red squares) in the left $(\boldsymbol{A})$ and right $(\boldsymbol{B})$ hemispheres using the ASD classifier.

racies in the range of $70.0-80.0 \%$. The classification $p$ value resulting from the permutation test was very low across, as well as within, modalities $(<0.004)$. The probability of obtaining specificity and sensitivity values higher than the ones obtained during cross-validation procedure by chance is thus extremely low.

A very similar profile of parameter importance was observed in the right hemisphere-despite it having generally lower classification accuracies. Here, individuals with ASD were correctly assigned to the appropriate diagnostic category in $65.0 \%$ of all cases (sensitivity $60.0 \%$, specificity $70.0 \%, p<0.03$ ) when all parameters were considered simultaneously (see Fig. $2 B, D$ ). As in the left hemisphere, cortical thickness as well as metric distortion displayed the best classification performance-although only cortical thickness measures reached statistical significance $(p<0.01)$. The discrepancy in overall classification accuracy between the two hemispheres was not due to differences in parameter variability, as there were no significant differences in parameter variance between homolog regions in different hemispheres (calculated across the whole sample) (see supplemental Fig. 1, available at www.jneurosci.org as supplemental material).

Thus, while all parameters provided statistically significant predictions in the left hemisphere, only the full model and cortical thickness displayed significant predictions in the right hemisphere. Highest classification accuracy (90.0\%) might thus be obtained by using cortical thickness measures exclusively in the left hemispheres.

Correlation coefficients between ADI-R subscales and the test margin coming from the combined model are listed in Table 4. The test margin in the left hemisphere was positively correlated with the ADI scores for individuals with ASD in the social $(r=$ $0.414, p<0.05)$ and communication domain $(r=0.62, p<$ $0.01)$. Therefore, individuals with higher values on these ADI subdomains are located on the extreme right relative on the hyperplane while the individuals with a lower level of impairment are mostly located in close proximity to the hyperplane overall. 

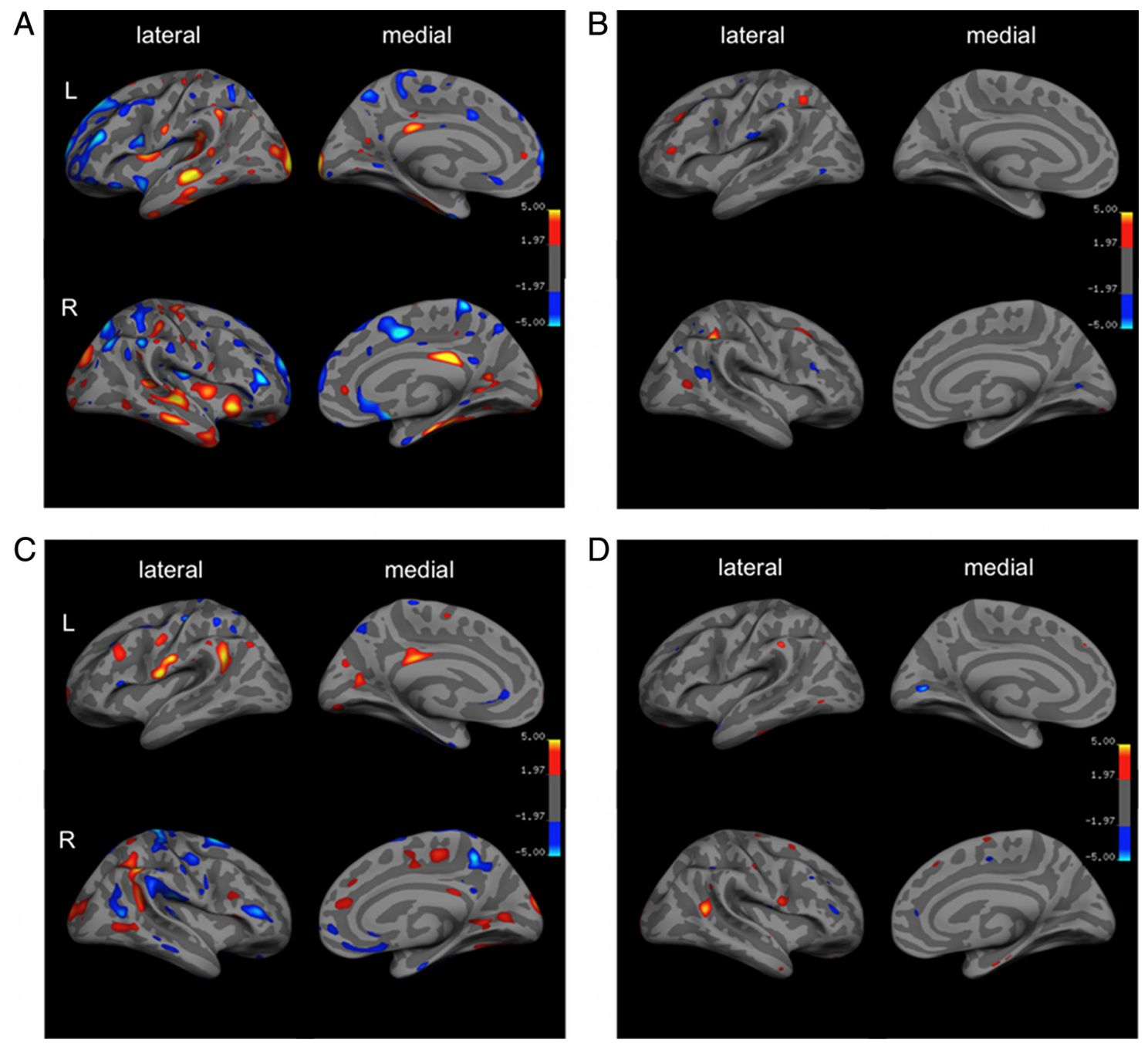

Figure 4. Discrimination maps for the five different morphometric features in the left $(\mathrm{L})$ and right $(\mathrm{R})$ hemispheres. Color maps represent the weight vector on the basis of the five modality classification for cortical thickness $(\boldsymbol{A})$, average convexity $(\boldsymbol{B})$, metric distortion $(\boldsymbol{C})$, and pial area $(\boldsymbol{D})$. Weights for the mean (radial) curvature did not exceed the set threshold. Positive weights (i.e., overall excess patters in ASD relative to controls) are displayed in red, and negative weights (i.e., overall deficit patterns in ASD relative to controls) are displayed in blue.

\section{Classification of individuals with ADHD using the established ASD classifier}

To establish the degree of clinical specificity of the discrimination algorithm to ASD, rather than to neurodevelopmental disorders in general, the established classifier was used to predict group membership of individuals with ADHD. To predict group membership, the model including all parameters was chosen as it provided best classification accuracy in the right hemisphere, and high accuracy in the left hemisphere. On the basis of the neuroanatomical information available for the left hemisphere, 15 out of the 19 individuals with ADHD were allocated to the control group (78.9\%), and 4 individuals with ADHD were allocated to the ASD group (21\%). Using the right hemisphere, the classifier allocated cases with ADHD with approximately equal frequencies ( $47 \%$ allocated to the ASD category; $52 \%$ allocated to the control category). The classification plots for individuals with ADHD are shown in Figure 3 for both hemispheres.

\section{Discrimination maps of ASD-specific abnormalities}

The spatial maps of brain regions identified as outlined above are shown in Figure 4 and a summary description can be found in Table 5. Each of these maps is a spatial representation of the SVM weight vector, and while they do not directly quantify the infor- mation content of each region, each map forms a spatially distributed pattern showing the relative contribution of each voxel to the decision function. Although "knock-out" techniques may be used to directly quantify the information content of particular regions, this approach may be hampered by substantial information redundancy across brain regions. Nonetheless, the spatial distribution of the weight vector does provide information about which brain regions contributed to classification and in this case is suggestive of a distributed pattern of relative deficit or excess in ASD with respect to controls. We emphasize that due to the multivariate character of SVM (i.e., it considers interregional correlations), each region in the discrimination maps should be interpreted in the context of the entire discriminating pattern and should not be considered in isolation. Further, individual regions may display high classification weights for several reasons, e.g., there is a large difference in volume between groups in that region, or the region is highly intercorrelated with other components of the network (i.e., pattern). This is of particular relevance to ASD, as individuals with the disorder most likely have abnormalities in the development of neural systems, in addition to differences in isolated regions. Thus, it is important to highlight that individual network components are not necessarily different 
Table 5. Regions displaying high discrimination weights between ASD and control group for volumetric measures

\begin{tabular}{|c|c|c|c|c|c|c|}
\hline Parameter & Region & Hemi & $x$ & $y$ & $z$ & $w$ \\
\hline \multicolumn{7}{|l|}{ Cortical thickness } \\
\hline \multirow[t]{14}{*}{ ASD $>$ controls } & Lateral orbitofrontal & $\mathrm{R}$ & 27 & 22 & -5 & 5.13 \\
\hline & Supramarginal gyrus & $\mathrm{L}$ & -49 & -45 & 35 & 4.37 \\
\hline & Superior parietal & $\mathrm{R}$ & 20 & -82 & 31 & 3.96 \\
\hline & Inferior temporal & $\mathrm{L}$ & -53 & -28 & -22 & 4.53 \\
\hline & Middle temporal & $L / R$ & \pm 48 & -28 & -7 & 6.66 \\
\hline & Superior temporal gyrus & $L / R$ & \pm 53 & -20 & -1 & 5.85 \\
\hline & Superior temporal sulcus & $\mathrm{R}$ & 51 & -34 & 7 & 4.08 \\
\hline & Parahippocampal gyrus & $L / R$ & \pm 31 & -27 & -16 & 3.27 \\
\hline & Fusiform gyrus & $\mathrm{L} / \mathrm{R}$ & -36 & -28 & -18 & 4.64 \\
\hline & Entorhinal cortex & $\mathrm{L} / \mathrm{R}$ & \pm 21 & -17 & -23 & 3.87 \\
\hline & Lateral occipital & $L / R$ & \pm 15 & -98 & 6 & 6.92 \\
\hline & Posterior cingulate gyrus & $\mathrm{L}$ & -7 & -33 & 28 & 3.97 \\
\hline & Anterior cingulate gyrus & $\mathrm{R}$ & -6 & 28 & -7 & -2.71 \\
\hline & Precuneus & $\mathrm{L}$ & -11 & -55 & 48 & -3.82 \\
\hline \multirow[t]{8}{*}{ ASD $<$ controls } & Rostral middle frontal & $L / R$ & \pm 35 & 36 & 9 & -5.60 \\
\hline & Superior frontal & $\mathrm{L} / \mathrm{R}$ & \pm 12 & 61 & 7 & -5.39 \\
\hline & Caudal middle frontal & $\mathrm{L}$ & -40 & 20 & 36 & -3.12 \\
\hline & Pars opercularis & $\mathrm{L}$ & & & & \\
\hline & Inferior parietal lobe & $\mathrm{R}$ & 41 & -60 & 36 & -3.95 \\
\hline & Superior parietal lobe & $\mathrm{R}$ & 17 & -64 & 50 & -5.00 \\
\hline & Precuneus & $\mathrm{R}$ & 13 & -37 & 63 & -3.18 \\
\hline & Anterior cingulate gyrus & $\mathrm{R}$ & 6 & 30 & -5 & -3.18 \\
\hline \multicolumn{7}{|l|}{ Surface area } \\
\hline \multirow[t]{6}{*}{ ASD $>$ controls } & Precentral & $\mathrm{R}$ & 56 & 5 & 15 & 3.23 \\
\hline & Orbitofrontal & $\mathrm{L}$ & -18 & -96 & -7 & 2.60 \\
\hline & Supramarginal gyrus & $\mathrm{L}$ & -55 & -40 & 36 & 3.45 \\
\hline & Inferior parietal & $\mathrm{R}$ & 49 & -50 & 12 & 4.57 \\
\hline & Inferior temporal lobe & $\mathrm{L}$ & -45 & -63 & -1 & 2.69 \\
\hline & Lateral occipital & $\mathrm{R}$ & 17 & -95 & 14 & 2.54 \\
\hline \multirow[t]{5}{*}{ ASD $<$ controls } & Superior frontal & $\mathrm{R}$ & 15 & 39 & 7 & -2.26 \\
\hline & Rostral middle frontal & $\mathrm{R}$ & 35 & 36 & 8 & -2.85 \\
\hline & Paracentral & $\mathrm{R}$ & 10 & -6 & 46 & -2.20 \\
\hline & Superior temporal & $\mathrm{L}$ & -51 & 5 & 13 & -3.98 \\
\hline & Pericalcarine fissure & $\mathrm{L}$ & -13 & -76 & 7 & -4.50 \\
\hline
\end{tabular}

Hemi, Hemisphere; L, left hemisphere; $R$, right hemisphere; $w$, weight of each cluster centroid.

between groups, but should be considered as constituent parts of a neuroanatomical network discriminating between groups.

As can be seen in this Figure 4, the five investigated parameters resulted in different spatially distributed patterns of regions with highest contribution to the discrimination. While cortical thickness provided best classification accuracy and maximum weight values, differences between groups were observed in both volumetric as well as geometric features.

\section{Cortical thickness}

In both hemispheres, the discriminative pattern for cortical thickness in ASD comprised regions in all four lobes of the cortex (Fig. 4A). Regional details are summarized in Table 5. The "excess pattern" (i.e., ASD > controls) comprised several temporal regions, including the right superior temporal sulcus [Brodmann area 22 (BA22)], medial and superior temporal gyrus (BA21/ BA22), and the parahippocampal gyrus (BA36), fusiform gyrus (BA20), and entorhinal cortex. In addition, the excess pattern included the inferior/superior parietal lobe (BA39), BA18 of the occipital lobe, and the anterior and posterior cingulate gyrus.

While the excess pattern comprised predominantly occipitotemporal regions, the pattern displaying a relative thinning of the cortex in ASD versus controls (i.e., "deficit pattern") included mainly frontal and parietal regions such as the middle frontal gyrus (BA46), the medial/superior frontal gyrus (BA10), and BA46 in the medial frontal gyrus. In addition, the deficit pattern contained the superior parietal cortex (BA40/7) and the anterior cingulate gyrus.

\section{Surface area}

Only a few surface-area (Fig. 4D) differences were observed in relatively small clusters with generally low weights. Details are summarized in Table 4.

\section{Regional geometric characteristics}

Apart from volumetric differences, features describing regional geometric characteristics also displayed high discrimination weights, which were summarized in Table 6. In both hemispheres, individuals with ASD displayed a pattern of relative increase in sulcal depth (Fig. $4 B$ ) in the intraparietal sulcus as well as in the superior frontal cortex. The discriminative pattern for cortical folding (Fig. 4C), as indicated by the metric distortion (i.e., Jacobian), included mainly bilateral parietal regions such as the inferior parietal lobe (BA39/40), and several regions of the right frontal lobe (e.g., supramarginal gyrus, postcentral gyrus, and orbitofrontal regions). In addition, the discriminative pattern in cortical folding comprised the precuneus.

\section{Morphometric profiles}

The discrimination maps show that different morphometric parameters elicited different spatial patterns of weights with some 
Table 6. Regions displaying high discriminative weights between ASD and control group for geometric measures

\begin{tabular}{|c|c|c|c|c|c|c|}
\hline Parameter & Region & Hemi & $x$ & $y$ & $z$ & $w$ \\
\hline \multicolumn{7}{|l|}{ Metric distortion } \\
\hline \multirow[t]{12}{*}{ ASD $>$ controls } & Precentral gyrus & $\mathrm{L}$ & -50 & -5 & 37 & 3.23 \\
\hline & Rostral middle frontal & $\mathrm{L}$ & -44 & 24 & 28 & 3.27 \\
\hline & Superior frontal gyrus & $\mathrm{R}$ & 14 & 40 & 14 & 2.51 \\
\hline & Supramarginal gyrus & $\mathrm{L}$ & -51 & -50 & 25 & 5.30 \\
\hline & Postcentral gyrus & $\mathrm{L}$ & -60 & -8 & 20 & 3.67 \\
\hline & Posterior cingulate gyrus & $\mathrm{L}$ & -7 & -28 & 29 & 3.50 \\
\hline & Pericalcarine fissure & $\mathrm{L}$ & -17 & -67 & 10 & 3.46 \\
\hline & Lateral occipital gyrus & $\mathrm{R}$ & 28 & -86 & 16 & 2.87 \\
\hline & Inferior parietal lobe & $\mathrm{R}$ & 47 & -45 & 37 & 4.90 \\
\hline & Middle temporal gyrus & $\mathrm{R}$ & 53 & -53 & 2 & 2.69 \\
\hline & Paracentral gyrus & $\mathrm{R}$ & 8 & -22 & 47 & 3.23 \\
\hline & Lingual gyrus & $\mathrm{R}$ & 25 & -55 & 2 & 2.50 \\
\hline \multirow[t]{9}{*}{ ASD $<$ controls } & Anterior cingulate & $\mathrm{L}$ & -6 & 34 & 0 & -2.75 \\
\hline & Postcentral gyrus & $\mathrm{R}$ & 30 & -28 & 63 & -3.98 \\
\hline & Supramarginal gyrus & $\mathrm{R}$ & 59 & -35 & 29 & -3.75 \\
\hline & Rostral middle frontal & $\mathrm{R}$ & 37 & 32 & 7 & -4.27 \\
\hline & Precentral gyrus & $\mathrm{R}$ & 42 & -6 & 47 & -3.59 \\
\hline & Middle temporal gyrus & $\mathrm{R}$ & 62 & -25 & -12 & -2.49 \\
\hline & Superior frontal lobe & $\mathrm{R}$ & 17 & 7 & 56 & -5.14 \\
\hline & Precuneus & $\mathrm{R}$ & 7 & -44 & 49 & -5.13 \\
\hline & Medial orbitofrontal gyrus & $\mathrm{R}$ & 7 & 32 & -147 & -2.36 \\
\hline \multicolumn{7}{|l|}{ Sulcal depth } \\
\hline \multirow[t]{4}{*}{ ASD $>$ controls } & Superior parietal lobe & $L / R$ & \pm 26 & -50 & 44 & 3.20 \\
\hline & Rostral middle frontal & $\mathrm{L}$ & -24 & 30 & 28 & 3.21 \\
\hline & Inferior parietal & $\mathrm{L}$ & 41 & -59 & 9 & 2.57 \\
\hline & Superior frontal & $L / R$ & 25 & 15 & 39 & 3.00 \\
\hline \multirow[t]{5}{*}{ ASD $<$ controls } & Supramarginal gyrus & $\mathrm{L}$ & -34 & -39 & 37 & -3.94 \\
\hline & Lateral occipital cortex & $\mathrm{L}$ & -45 & -63 & 0 & -2.85 \\
\hline & Inferior parietal & $\mathrm{R}$ & 48 & -52 & 17 & -2.76 \\
\hline & Rostral middle frontal & $\mathrm{R}$ & 40 & 25 & 18 & -2.92 \\
\hline & Pericalcarine fissure & $\mathrm{R}$ & 18 & -70 & 12 & -2.83 \\
\hline
\end{tabular}

Hemi, Hemisphere; L, left hemisphere; $R$, right hemisphere; $w$, weight of each cluster centroid.

A

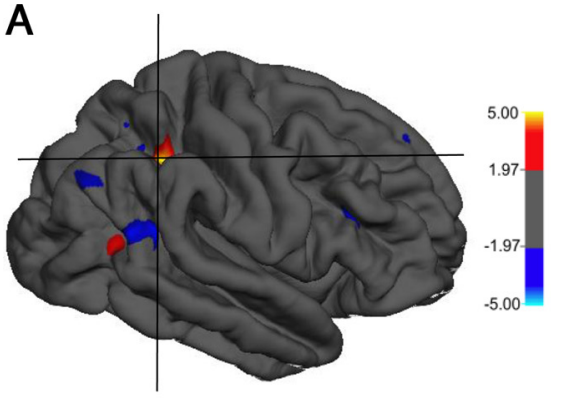

C

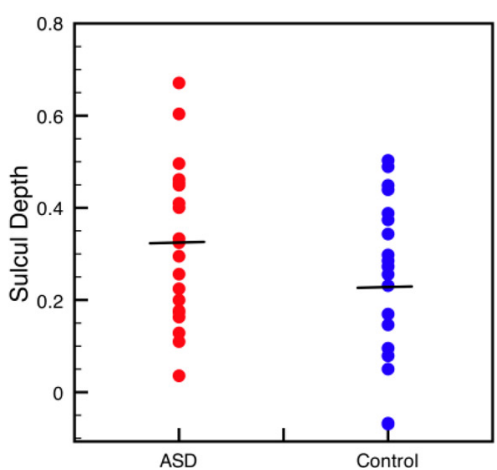

B

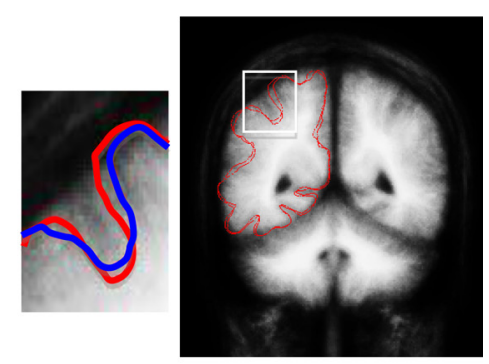

D

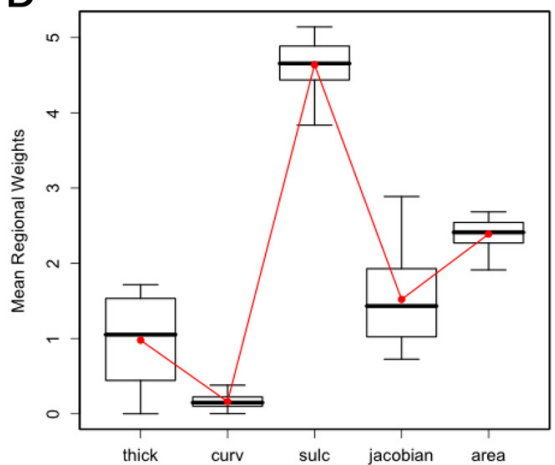

Figure 5. Visualization of the morphometric abnormalities in the right intraparietal sulcus. Color maps represent the weight vector score (A). B, Outlines of the cortical surface for ASD (red) and control (blue) group. This main discriminating factor in this group was an increase in sulcal depth in ASD relative to controls. Differences in sulcal depth for this ROI are shown for both groups in C. D, Morphometric profile for this region. Profiles were derived by averaging the weight vector scores across vertices within this region of interest, and for the different morphometric parameters. Weights were identified on the basis of the concatenated SVM model, thus showing the relative contribution of parameters in this ROI.

overlap between particular parameter pairs. The weights for individual parameters are thus not uniform across the cortex but vary from region to region (i.e., vertex to vertex). This can also be seen on the basis of the morphometric profile for individual regions, which are displayed in Figures $5 D$, $6 D, 7 D$, and $8 C$ and listed in Table 7 . These profiles visualize the mean parameter weights for individual features within regions of interest.

In the intraparietal sulcus for instance, high discrimination weights were observed for differences in sulcal depth with lower weights for volumetric features such as cortical thickness or surface area (see Fig. 5). A similar profile was also seen in the inferior parietal lobe (BA39). Here, the weights for volumetric parameters such as cortical thickness or surface area were very low in comparison to the weights associated with the pattern of cortical folding (i.e., metric distortion) (see Fig. 6).

Other regions, however, displayed high weights for volumetric parameters exclusively. For instance, in the temporal sulcus (BA21), high weights were observed for cortical thickness exclusively in the light of low weights in all other parameters (see Fig. 8). There were also regions 
with equally high weights in volumetric as well as geometric features-e.g., the anterior cingulate gyrus. Here, cortical thickness as well as local cortical folding displayed high parameter weights.

In summary, we observed a spatially distributed pattern of regions that can be used to differentiate individuals with ASD from controls. Overall, highest weights were observed for measures of cortical thickness, followed by geometric features such as sulcal depth and metric distortion, which were predominantly observed in parietal regions. This suggests that both volumetric and geometric features play an important role in distinguishing between ASD and control group. Last we demonstrated that the parameter weights for individual cortical features were region dependent.

\section{Discussion}

Autism affects multiple aspects of the cerebral anatomy, which makes its neuroanatomical correlates inherently difficult to describe. Here, we used a multiparameter classification approach to characterize the complex and subtle gray matter differences in adults with ASD. SVM achieved good separation between groups, and revealed spatially distributed and largely nonoverlapping patterns of regions with highest classification weights for each of five morphological features. Our results confirm that the neuroanatomy of ASD is truly multidimensional affecting multiple neural systems. The discriminating patterns detected using SVM may help further exploration of the genetic and neuropathological underpinnings of ASD.

There is good evidence to suggest that several aspects of cerebral morphology are implemented in ASD_including both volumetric and geometric features (Levitt et al., 2003; Nordahl et al., 2007). However, these are normally explored in isolation. Here, we aimed to establish a framework for multiparameter image classification to describe differences in gray matter neuroanatomy in autism in multiple dimensions, and to explore the predictive power of individual parameters for group membership. This was achieved using a multiparameter classifier incorporating volumetric and geometric features at each cerebral vertex. In the left hemisphere, SVM correctly classified $85.0 \%$ of all cases overall at a sensitivity and specificity as high as $90.0 \%$ and $80.0 \%$, respectively, using all five morphological features. This level of sensitivity compares well with behaviorally guided diagnostic tools whose accuracies are on average $\sim 80 \%$. Naturally, one would ex-

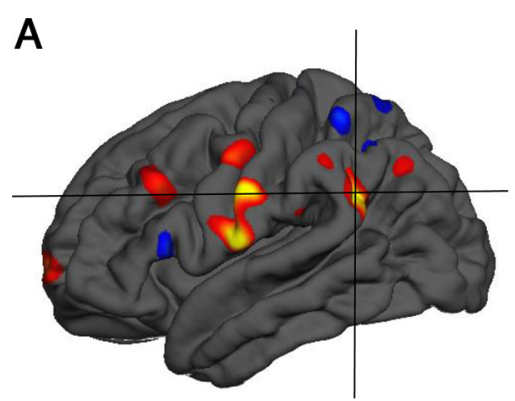

B
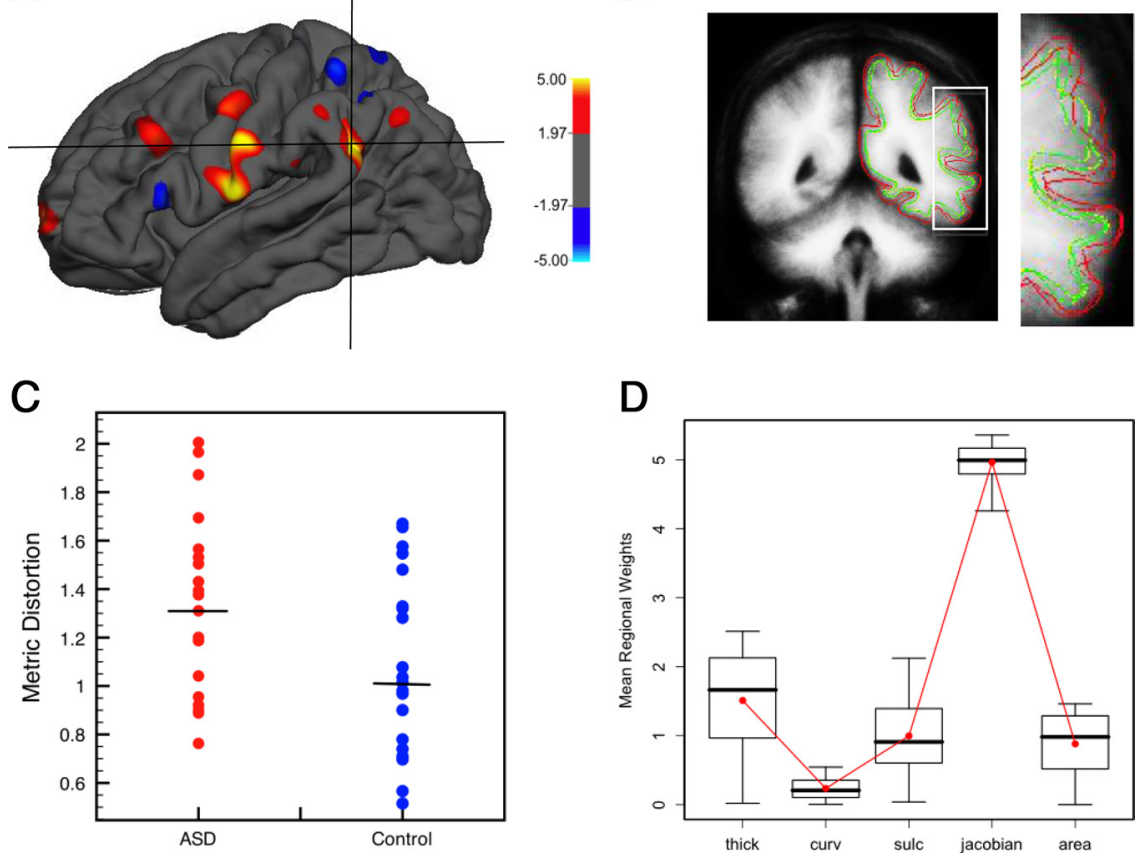

Figure 6. $\boldsymbol{A}$, Visualization of the morphometric abnormalities in the left inferior parietal lobe (BA39). $\boldsymbol{B}, 0$ utlines of the cortical surface for ASD and control group. Differences in metric distortion for this ROI are shown for both groups in C. D, Morphometric profile (see Fig. 4 legend).
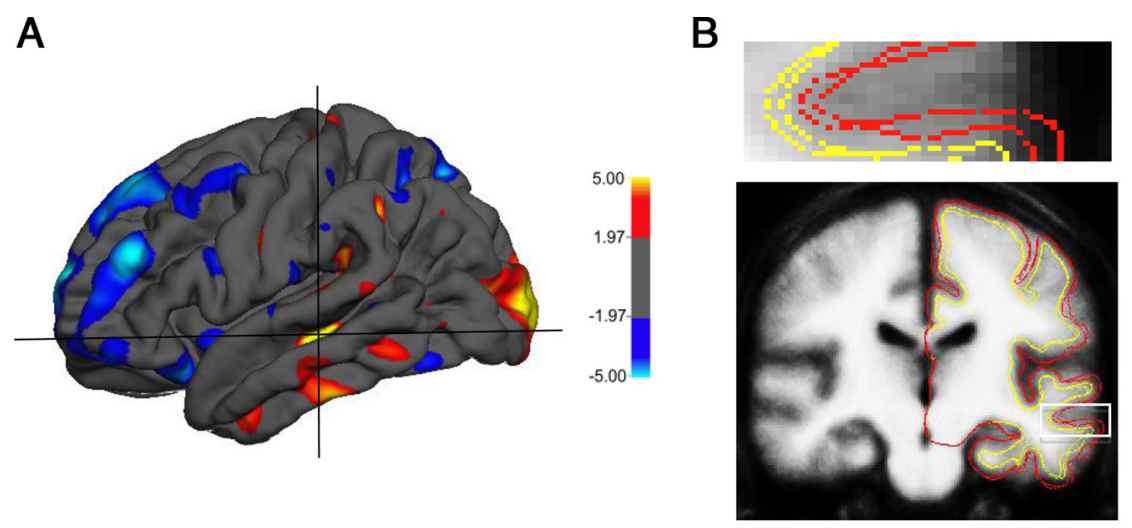

C

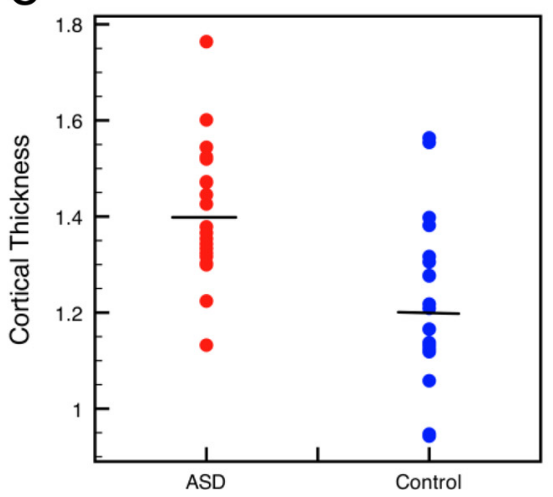

D

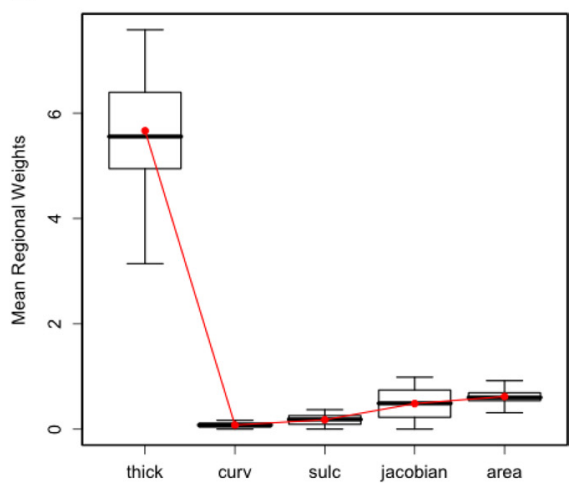

Figure 7. $\quad \boldsymbol{A}$, Morphometric abnormalities in the middle temporal sulcus. $\boldsymbol{B}$, Visualization of cortical thickness measures for ASD (red straight line) and control (blue straight line) group. In this region cortical thickness exclusively discriminated between groups with individuals with ASD displaying increased thickness relative to controls $(\boldsymbol{C}, \boldsymbol{D})$. 
A
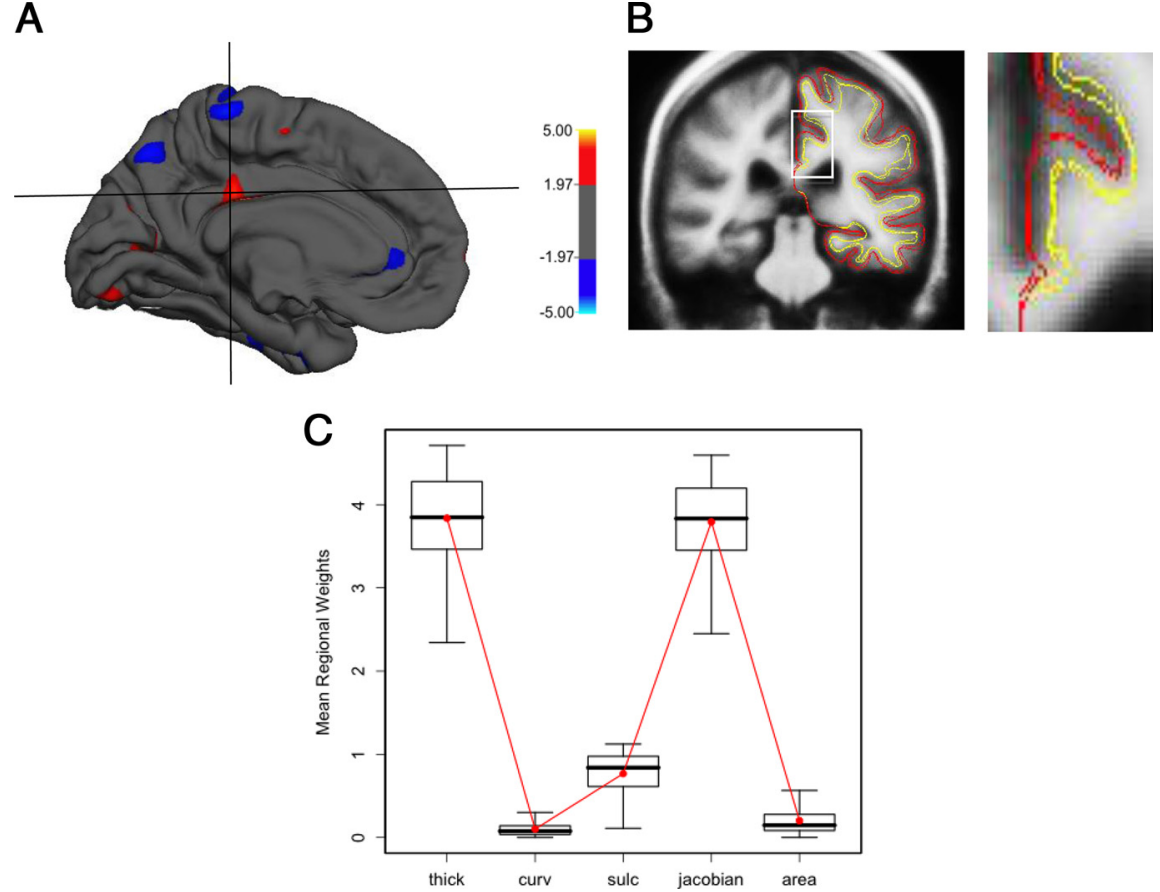

Figure 8. $A$, Morphometric abnormalities in the posterior cingulate gyrus. $\boldsymbol{B}, \boldsymbol{C}$, Here, a combination of cortical thickness and folding pattern led to a high contribution to the classification in that region.

Table 7. Mean discrimination weights within regions of interest for individual morphometric features

\begin{tabular}{llllll}
\hline & \multicolumn{3}{l}{$\operatorname{Mean}(w)$} & & \\
\cline { 2 - 6 } Region & $\begin{array}{l}\text { Cortical } \\
\text { thickness }\end{array}$ & $\begin{array}{l}\text { Mean } \\
\text { curvature }\end{array}$ & $\begin{array}{l}\text { Sulcal } \\
\text { depth }\end{array}$ & $\begin{array}{l}\text { Metric } \\
\text { distortion }\end{array}$ & $\begin{array}{l}\text { Surface } \\
\text { area }\end{array}$ \\
\hline Intraparietal sulcus (R) & 0.97 & 0.16 & 4.63 & 1.51 & 2.38 \\
Inferior parietal lobe (L) & 1.51 & 0.23 & 0.99 & 4.96 & 0.88 \\
Medial temporal sulcus (L) & 5.66 & 0.07 & 0.17 & 0.48 & 0.62 \\
Posterior cingulate gyrus (L) & 3.83 & 0.09 & 0.78 & 3.79 & 0.20 \\
\hline Pinnyyyy
\end{tabular}

$R$, Right hemisphere; L, left hemisphere; $w$, weight.

pect lower sensitivity values than the test used for defining the "autistic prototype" itself (i.e., ADI-R). Thus, if a classifier is trained on the basis of true positives identified by diagnostic tools, the maximal classification accuracy that could be reached is only as good as the measurements used to identify true positives.

The significant predictive value of pattern classification approaches may have potential clinical applications. Currently, ASD is diagnosed solely on the basis of behavioral criteria. The behavioral diagnosis is however often time consuming and can be problematic, particularly in adults. Also, different biological etiologies might result in the same behavioral phenotype [the "autisms" (Geschwind, 2007)], which is undetectable using behavioral measures alone. Thus, the existence of an ASD biomarker such as brain anatomy might be useful to facilitate and guide the behavioral diagnosis. This would, however, require further extensive exploration in the clinical setting, particularly with regards to classifier specificity to ASD rather than neurodevelopmental conditions in general.

To address the issue of clinical specificity, the established ASD classifier was used to classify individuals with ADHD-a neurodevelopmental control group. Bilaterally, the ASD classifier did not allocate the majority of ADHD subjects to the ASD category. This indicates that it does not perform equally well for other neurodevelopmental conditions, and is more specific to ASD. To further demonstrate that the classification is driven by autistic symptoms, the test margins of individuals with ASD were correlated with measures of symptom severity (Ecker et al., 2010). We found that larger margins were associated with more severe impairments in the social and communication domain of the ADI-R. The classifier therefore seems to use neuroanatomical information specifically related to ASD rather than simply reflecting nonspecific effects introduced by any kind of pathology. However, due to a recent scanner upgrade, ADHD scans were acquired with different acquisition parameters, while manufacturer, field strength, and pulse sequence remained the same. FreeSurfer has been demonstrated to show good test-retest reliability particularly within scannermanufacturer and field strength (Han et al., 2006), but we cannot exclude the possibility that systematic differences in regional contrast may have affected the ADHD classification. Future research is thus needed to validate the $\mathrm{ADHD}$ findings on an independent sample.

The overall classification accuracy varied across hemispheres $(79.0 \%$ left vs $65.0 \%$ right) in the absence of interhemispheric differences in parameter variability. Hemisphere laterality is an area, which remains relatively unexplored in autism. While our data suggest that the left hemisphere is better at discriminating between groups (i.e., is more "abnormal"), it is unclear whether this discrepancy is due to quantitative differences in parameters or to qualitative aspects of the discriminating patterns (i.e., additional regions). Furthermore, it is also not possible to identify whether individuals with ASD display a higher (lower) degree of cortical asymmetry relative to controls. There is some evidence to suggest that individuals with ASD show a lower degree of "leftward" (i.e., left > right) cortical symmetry than controls (Herbert et al., 2005), which may explain differences in classification accuracy. There is also evidence to suggest that the left hemisphere is under tighter genetic control than the right hemisphere (Thompson et al., 2001), which may be of relevance to a highly heritable condition such as ASD. However, a direct numerical comparison between hemispheres is needed to address this issue directly.

The classification accuracy not only varied across hemispheres but also across morphometric parameters. Bilaterally, cortical thickness provided the best classification accuracy and highest regional weights. Differences in cortical thickness have been reported previously in ASD for both increases (Chung et al., 2005; Hardan et al., 2006) as well as decreases (Chung et al., 2005; Hadjikhani et al., 2006), and in similar regions as reported here (i.e., parietal, temporal, and frontal areas). The overlap with previous studies indicates that these regions display high classification weights due to a quantitative (i.e., "true") difference rather than high intercorrelations with thickness measures in other brain regions.

Certain geometric features such as average convexity and metric distortion provided above chance classifications as well, particularly in parietal, temporal, and frontal regions, and in areas of the cingulum. Average convexity and metric distortion measure different aspects of cortical geometry (see Materials and Meth- 
ods) and have previously been linked to ASD, as has sulcal depth (Nordahl et al., 2007). Such geometric features were suggested to reflect abnormal patterns of cortical connectivity. There have also been reports of abnormal patterns of gyrification (Piven et al., 1990; Hardan et al., 2004) and large-scale displacements of the major sulci (Levitt et al., 2003). Thus, our study provides further evidence to support the hypothesis that the "autistic brain" is not just bigger or smaller but is also abnormally shaped.

While we demonstrated that the neuroanatomy of ASD is multidimensional, the etiology of such multivariate differences remains unclear. Here, little or no spatial overlap was observed between the discriminating patterns for individual parameters. Such region dependency was also observed in the regional morphometric profiles displaying the distribution of weights across multiple cortical features in a region of interest. If one assumes that different cortical features reflect different neuropathological processes, such region- and parameter-dependent variations may reflect the multifactorial etiology of ASD. For example, evidence suggests that cortical thickness and surface area reflect different neurobiological processes and are associated with different genetic mechanisms (Panizzon et al., 2009). Cortical thickness is likely to reflect dendritic arborization (Huttenlocher, 1990) or changing myelination at the gray/white matter interface (Sowell et al., 2004). In contrast, surface area is influenced by the division of progenitor cells in the embryological periventricular area, and is associated with the number of minicolumns (Rakic, 1988). Instead, geometric differences are predominantly linked with the development of neuronal connections and cortical pattern of connectivity, and are thus a marker for cerebral development (Armstrong et al., 1995; Van Essen, 1997). It is therefore likely that the reported maps reflect multiple genetic and/or neurobiological etiologies, which need further investigation. Thus, our findings should be interpreted in the context of a number of methodological limitations.

First, the classification algorithm is highly specific to the particular sample used for "training" the classifier, namely highfunctioning adults with ASD. The advantage of this approach is that the classifier offers high specificity with regard to this particular subject group, but is less specific to other cohorts on the spectrum. Due to the small sample size, it was also not possible to reliably investigate differences between high-functioning autism and Asperger's syndrome. Evidence (Howlin, 2003) suggests that by adulthood these groups are largely indistinguishable at the phenotypic level. However, the extent to which these groups differ at the level of brain anatomy is unknown, and may be investigating using SVM in the future. Second, $85 \%$ of ASD participants in our sample were diagnosed using the ADI-R, and $15 \%$ were diagnosed using the ADOS. As both diagnostic tools measure autistic symptoms at different developmental stages, the classifier may be biased toward individuals with an early diagnosis of ASD. Although it is not expected that classifier performance on the basis of ADOS and ADI differ drastically, diagnostic heterogeneity may be a potential limitation. Last, SVM is a multivariate technique and hence offers a limited degree of interpretability of specific network components. Additional analysis such as "searchlight" or "virtual lesions" approaches (Averbeck et al., 2006; Kriegeskorte et al., 2006; Pessoa and Padmala, 2007) may therefore be combined with SVM in the future to establish the relative contribution of individual regions/parameters to the overall classification performance.

Nevertheless, while classification values and specific patterns we report must be considered as preliminary, our study offers a "proof of concept" for describing the complex multidimensional gray matter differences in ASD.

\section{References}

Amaral DG, Schumann CM, Nordahl CW (2008) Neuroanatomy of autism. Trends Neurosci 31:137-145.

Armstrong E, Schleicher A, Omran H, Curtis M, Zilles K (1995) The ontogeny of human gyrification. Cereb Cortex 5:56-63.

Averbeck BB, Latham PE, Pouget A (2006) Neural correlations, population coding and computation. Nat Rev Neurosci 7:358-366.

Barkley RA (2006) Attention-deficit hyperactivity disorder-a handbook for diagnosis and treatment, Ed 3. New York: Guilford.

Burges CJC (1998) A tutorial on support vector machines for pattern recognition. Data Min Knowl Discov 2:121-167.

Chung MK, Robbins S, Evans AC (2005) Unified statistical approach to cortical thickness analysis. Inf Process Med Imaging 19:627-638.

Dale AM, Fischl B, Sereno MI (1999) Cortical surface-based analysis. I. Segmentation and surface reconstruction. Neuroimage 9:179-194.

Davatzikos C, Fan Y, Wu X, Shen D, Resnick SM (2008) Detection of prodromal Alzheimer's disease via pattern classification of magnetic resonance imaging. Neurobiol Aging 29:514-523.

Desikan RS, Ségonne F, Fischl B, Quinn BT, Dickerson BC, Blacker D, Buckner RL, Dale AM, Maguire RP, Hyman BT, Albert MS, Killiany RJ (2006) An automated labeling system for subdividing the human cerebral cortex on MRI scans into gyral based regions of interest. Neuroimage 31:968-980.

Ecker C, Rocha-Rego V, Johnston P, Mourao-Miranda J, Marquand A, Daly EM, Brammer MJ, Murphy C, Murphy DG, MRC AIMS Consortium (2010) Investigating the predictive value of whole-brain structural MR scans in autism: a pattern classification approach. Neuroimage 49:44-56.

Fischl B, Dale AM (2000) Measuring the thickness of the human cerebral cortex from magnetic resonance images. Proc Natl Acad Sci U S A 97:11050-11055.

Fischl B, Sereno MI, Dale AM (1999) Cortical surface-based analysis. II: Inflation, flattening, and a surface-based coordinate system. Neuroimage 9:195-207.

Fischl B, Liu A, Dale AM (2001) Automated manifold surgery: constructing geometrically accurate and topologically correct models of the human cerebral cortex. IEEE Trans Med Imaging 20:70-80.

Fischl B, Salat DH, Busa E, Albert M, Dieterich M, Haselgrove C, van der Kouwe A, Killiany R, Kennedy D, Klaveness S, Montillo A, Makris N, Rosen B, Dale AM (2002) Whole brain segmentation: automated labeling of neuroanatomical structures in the human brain. Neuron 33:341-355.

Fischl B, Salat DH, van der Kouwe AJ, Makris N, Ségonne F, Quinn BT, Dale AM (2004a) Sequence-independent segmentation of magnetic resonance images. Neuroimage 23 [Suppl 1]:S69-S84.

Fischl B, van der Kouwe A, Destrieux C, Halgren E, Ségonne F, Salat DH, Busa E, Seidman LJ, Goldstein J, Kennedy D, Caviness V, Makris N, Rosen B, Dale AM (2004b) Automatically parcellating the human cerebral cortex. Cereb Cortex 14:11-22.

Geschwind D (2007) Autism: searching for coherence. Biol Psychiatry 62:949-950.

Geschwind DH, Levitt P (2007) Autism spectrum disorders: developmental disconnection syndromes. Curr Opin Neurobiol 17:103-111.

Hadjikhani N, Joseph RM, Snyder J, Tager-Flusberg H (2006) Anatomical differences in the mirror neuron system and social cognition network in autism. Cereb Cortex 16:1276-1282.

Han X, Jovicich J, Salat D, van der Kouwe A, Quinn B, Czanner S, Busa E, Pacheco J, Albert M, Killiany R, Maguire P, Rosas D, Makris N, Dale A, Dickerson B, Fischl B (2006) Reliability of MRI-derived measurements of human cerebral cortical thickness: the effects of field strength, scanner upgrade and manufacturer. Neuroimage 32:180-194.

Hardan AY, Jou RJ, Keshavan MS, Varma R, Minshew NJ (2004) Increased frontal cortical folding in autism: a preliminary MRI study. Psychiatry Res 131:263-268

Hardan AY, Muddasani S, Vemulapalli M, Keshavan MS, Minshew NJ (2006) An MRI study of increased cortical thickness in autism. Am J Psychiatry 163:1290-1292.

Herbert MR, Ziegler DA, Deutsch CK, O’Brien LM, Kennedy DN, Filipek PA, Bakardjiev AI, Hodgson J, Takeoka M, Makris N, Caviness VS Jr (2005) 
Brain asymmetries in autism and developmental language disorder: a nested whole-brain analysis. Brain 128:213-226.

Howlin P (2003) Outcome in high-functioning adults with autism with and without early language delays: implications for the differentiation between autism and Asperger syndrome. J Autism Dev Disord 33:3-13.

Huttenlocher PR (1990) Morphometric study of human cerebral cortex development. Neuropsychologia 28:517-527.

Jovicich J, Czanner S, Greve D, Haley E, van der Kouwe A, Gollub R, Kennedy D, Schmitt F, Brown G, Macfall J, Fischl B, Dale A (2006) Reliability in multi-site structural MRI studies: effects of gradient non-linearity correction on phantom and human data. Neuroimage 30:436-443.

Klöppel S, Stonnington CM, Chu C, Draganski B, Scahill RI, Rohrer JD, Fox NC, Jack CR Jr, Ashburner J, Frackowiak RS (2008) Automatic classification of MR scans in Alzheimer's disease. Brain 131:681-689.

Kriegeskorte N, Goebel R, Bandettini P (2006) Information-based functional brain mapping. Proc Natl Acad Sci U S A 103:3863-3868.

Kuperberg GR, Broome MR, McGuire PK, David AS, Eddy M, Ozawa F, Goff D, West WC, Williams SC, van der Kouwe AJ, Salat DH, Dale AM, Fischl B (2003) Regionally localized thinning of the cerebral cortex in schizophrenia. Arch Gen Psychiatry 60:878-888.

Levitt JG, Blanton RE, Smalley S, Thompson PM, Guthrie D, McCracken JT, Sadoun T, Heinichen L, Toga AW (2003) Cortical sulcal maps in autism. Cereb Cortex 13:728-735.

Lord C, Rutter M, Goode S, Heemsbergen J, Jordan H, Mawhood L, Schopler E (1989) Autism Diagnostic Observation Schedule: a standardized observation of communicative and social behavior. J Autism Dev Disord 19:185-212.

Lord C, Rutter M, Le Couteur A (1994) Autism Diagnostic Interview-Revised: a revised version of a diagnostic interview for caregivers of individuals with possible pervasive developmental disorders. J Autism Dev Disord 24:659-685.

McAlonan GM, Daly E, Kumari V, Critchley HD, van Amelsvoort T, Suckling J, Simmons A, Sigmundsson T, Greenwood K, Russell A, Schmitz N, Happe F, Howlin P, Murphy DG (2002) Brain anatomy and sensorimotor gating in Asperger's syndrome. Brain 125:1594-1606.

McAlonan GM, Cheung V, Cheung C, Suckling J, Lam GY, Tai KS, Yip L, Murphy DG, Chua SE (2005) Mapping the brain in autism. A voxelbased MRI study of volumetric differences and intercorrelations in autism. Brain 128:268-276.

Mourão-Miranda J, Bokde AL, Born C, Hampel H, Stetter M (2005) Classifying brain states and determining the discriminating activation patterns: support vector machine on functional MRI data. Neuroimage 28:980-995.

Mourão-Miranda J, Reynaud E, McGlone F, Calvert G, Brammer M (2006) The impact of temporal compression and space selection on SVM analysis of single-subject and multi-subject fMRI data. Neuroimage 33:1055-1065.

Nordahl CW, Dierker D, Mostafavi I, Schumann CM, Rivera SM, Amaral DG, Van Essen DC (2007) Cortical folding abnormalities in autism revealed by surface-based morphometry. J Neurosci 27:11725-11735.

Panizzon MS, Fennema-Notestine C, Eyler LT, Jernigan TL, Prom-Wormley E, Neale M, Jacobson K, Lyons MJ, Grant MD, Franz CE, Xian H, Tsuang M, Fischl B, Seidman L, Dale A, Kremen WS (2009) Distinct genetic influences on cortical surface area and cortical thickness. Cereb Cortex 19:2728-2735.
Pessoa L, Padmala S (2007) Decoding near-threshold perception of fear from distributed single-trial brain activation. Cereb Cortex 17:691-701.

Piven J, Berthier ML, Starkstein SE, Nehme E, Pearlson G, Folstein S (1990) Magnetic resonance imaging evidence for a defect of cerebral cortical development in autism. Am J Psychiatry 147:734-739.

Rakic P (1988) Defects of neuronal migration and the pathogenesis of cortical malformations. Prog Brain Res 73:15-37.

Rojas DC, Peterson E, Winterrowd E, Reite ML, Rogers SJ, Tregellas JR (2006) Regional gray matter volumetric changes in autism associated with social and repetitive behavior symptoms. BMC Psychiatry 6:56.

Rosas HD, Liu AK, Hersch S, Glessner M, Ferrante RJ, Salat DH, van der Kouwe A, Jenkins BG, Dale AM, Fischl B (2002) Regional and progressive thinning of the cortical ribbon in Huntington's disease. Neurology 58:695-701.

Salat DH, Buckner RL, Snyder AZ, Greve DN, Desikan RS, Busa E, Morris JC, Dale AM, Fischl B (2004) Thinning of the cerebral cortex in aging. Cereb Cortex 14:721-730.

Schoelkopf M, Smola AJ (2002) Learning with Kernes. Cambridge, MA: MIT Press.

Ségonne F, Dale AM, Busa E, Glessner M, Salat D, Hahn HK, Fischl B (2004) A hybrid approach to the skull stripping problem in MRI. Neuroimage 22:1060-1075.

Ségonne F, Pacheco J, Fischl B (2007) Geometrically accurate topologycorrection of cortical surfaces using nonseparating loops. IEEE Trans Med Imaging 26:518-529.

Sowell ER, Thompson PM, Leonard CM, Welcome SE, Kan E, Toga AW (2004) Longitudinal mapping of cortical thickness and brain growth in normal children. J Neurosci 24:8223-8231.

Thompson PM, Cannon TD, Narr KL, van Erp T, Poutanen VP, Huttunen M, Lönnqvist J, Standertskjöld-Nordenstam CG, Kaprio J, Khaledy M, Dail R, Zoumalan CI, Toga AW (2001) Genetic influences on brain structure. Nat Neurosci 4:1253-1258.

Toal F, Murphy DG, Murphy KC (2005) Autistic-spectrum disorders: lessons from neuroimaging. Br J Psychiatry 187:395-397.

Van Essen DC (1997) A tension-based theory of morphogenesis and compact wiring in the central nervous system. Nature 385:313-318.

Vapnik VN (1995) The nature of statistical learning theory. New York: Springer.

Waiter GD, Williams JH, Murray AD, Gilchrist A, Perrett DI, Whiten A (2004) A voxel-based investigation of brain structure in male adolescents with autistic spectrum disorder. Neuroimage 22:619-625.

Ward MF, Wender PH, Reimherr FW (1993) The Wender Utah Rating Scale: an aid in the retrospective diagnosis of childhood attention deficit hyperactivity disorder. Am J Psychiatry 150:885-890.

Wechsler D (1999) Wechsler Abbreviated Scale of Intelligence (WASI). San Antonio, TX: Harcourt Assessment.

Wisco JJ, Kuperberg G, Manoach D, Quinn BT, Busa E, Fischl B, Heckers S, Sorensen AG (2007) Abnormal cortical folding patterns within Broca's area in schizophrenia: evidence from structural MRI. Schizophr Res 94:317-327.

World Health Organization (1992) International statistical classification of diseases and health related problems - 10th revision. 